\title{
Single-shooting homotopy method for parameter identification in dynamical systems
}

\author{
C. P. Vyasarayani, Thomas Uchida, and John McPhee \\ Department of Systems Design Engineering, University of Waterloo, 200 University Avenue West, Waterloo, Ontario, N2L 3G1, Canada
}

(Received 31 May 2011; published 2 March 2012)

\begin{abstract}
An algorithm for identifying parameters in dynamical systems is developed in this work using homotopy transformations and the single-shooting method. The equations governing the dynamics of the mathematical model are augmented with observer-like homotopy terms that smooth the objective function. As a result, premature convergence to a local minimum is avoided and the obtained parameter estimates are globally optimal. Numerical examples are presented to demonstrate the application of the proposed approach to chaotic systems.
\end{abstract}

DOI: 10.1103/PhysRevE.85.036201

PACS number(s): 05.45.Tp, 02.30.Hq

\section{INTRODUCTION}

The identification of parameters in dynamical systems is a challenging problem $[1,2]$. In this work, we focus specifically on problems where the functional form of the system of differential equations is known, as studied in [3-7]. In the context of deterministic global optimization [8], the present work focuses on the development of a new theory using homotopy optimization to obtain globally optimal parameters in systems governed by nonlinear ordinary differential equations (ODEs). While the theory and results presented herein are applicable to any dynamical system governed by ODEs, we demonstrate the application of our theory to chaotic systems. Since chaotic systems are highly sensitive to both initial conditions and system parameters [9], they represent challenging test cases for any parameter identification algorithm.

One of two fundamental strategies is commonly employed in parameter identification: synchronization or optimization. The former involves coupling to the mathematical model an auxiliary system that governs the dynamics of the parameters [5-7,10]. Convergence of the parameters to the actual values is achieved once the mathematical model has synchronized with the experimental data. The theory of Lyapunov stability can be used to study the convergence characteristics of such a system [11]. The conditions under which synchronization will occur in nonlinear systems can be found in the work of Letellier and Aguirre [12].

The parameter identification problem is even more challenging if the system is unobservable - that is, if only partial state measurements are obtained experimentally and are insufficient in number or richness to recover the remaining system states. In such cases, an optimization approach $[13,14]$ is required, of which there are two varieties: single-shooting and multiple-shooting, the latter of which can be implemented with $[3,4]$ or without [14] penalty terms. Provided the conditions of parameter identifiability [15] are met, it is possible to recover the system parameters using only partial state measurements; however, it might not be possible to recover the unknown initial conditions of unmeasured states. Single-shooting algorithms involve repeatedly simulating a mathematical model of the physical system for the same duration as the experiment, comparing the simulated response to the measured response and updating the parameter estimates after each simulation. In multiple-shooting algorithms, the experimental data are divided into several shorter fragments, each of which is used in a separate single-shooting procedure. Constraints ensure that the final states of each fragment are sufficiently close to the initial states of the fragment that follows. By performing shorter simulations, multiple-shooting algorithms are less likely to diverge when identifying parameters in chaotic systems.

In the optimization step, the objective function being minimized is typically defined as the Euclidean distance between the experimental data and the simulated response. Since the objective function generally contains many local minima, local search methods (e.g., Gauss-Newton, gradient descent, Levenberg-Marquardt, Nelder-Mead) must be used with caution, since they are likely to converge to these local minima. Recently, stochastic optimization techniques [16,17] have been used for their ability to find a global minimum in the presence of local minima; however, stochastic optimization generally requires a large number of iterations and is, therefore, time-consuming.

In this paper, the idea of homotopy optimization [18-20] is applied to dynamical systems and, in particular, those that are chaotic in nature. By augmenting the system equations with observer-like terms, the homotopy optimization technique avoids diverging when simulating chaotic systems, but does so using single-shooting rather than the more complicated multiple-shooting approach. Furthermore, homotopy optimization avoids converging to a local minimum without resorting to stochastic techniques. As will be demonstrated, this approach is suitable for identifying parameters in dynamical and chaotic systems. This paper extends the previous homotopy work of the authors [21,22], and also demonstrates that the objective function becomes quadratic with the introduction of the homotopy transformation.

\section{MATHEMATICAL MODELING}

Suppose the parameters $\mathbf{p}$ that minimize a given function $\mathcal{F}(\mathbf{p})$ are being sought. In the homotopy optimization approach, a new function is defined as follows:

$$
\mathcal{H}(\mathbf{p}, \lambda)=(1-\lambda) \mathcal{F}(\mathbf{p})+\lambda \mathcal{G}(\mathbf{p}),
$$

where $\mathcal{G}(\mathbf{p})$ is a convex function whose global minimum is known. The arguments that minimize $\mathcal{H}(\mathbf{p}, \lambda)$ at a given value of $\lambda$ shall be referred to herein as ${ }^{\lambda} \mathbf{p}^{*}$. The optimization procedure begins with $\lambda=1$, where the global minimum of $\mathcal{H}\left({ }^{1} \mathbf{p}^{*}, 1\right)=\mathcal{G}\left({ }^{1} \mathbf{p}^{*}\right)$ is known by design. The value of $\lambda$ is then decreased by a small amount $\delta \lambda$, and the function $\mathcal{H}(\mathbf{p}, 1-\delta \lambda)$ 
is minimized using ${ }^{1} \mathbf{p}^{*}$ as the initial guess. Once the optimal parameters ${ }^{1-\delta \lambda} \mathbf{p}^{*}$ have been obtained, $\lambda$ is further decreased to $1-2 \delta \lambda$ and the minimization is repeated, again using as an initial guess the optimal parameter vector corresponding to the previous value of $\lambda$. This process is repeated until $\lambda=0$ and the original optimization problem is recovered-that is, $\mathcal{H}(\mathbf{p}, 0)=\mathcal{F}(\mathbf{p})$. An important condition for convergence is the continuity of ${ }^{\lambda} \mathbf{p}^{*}$ between the global minimum of $\mathcal{G}(\mathbf{p})$ and that of $\mathcal{F}(\mathbf{p})$.

The differential equations governing the dynamics of an experimental system are assumed to be of the following general form:

$$
\dot{\mathbf{q}}_{e}=\mathbf{f}\left(\mathbf{q}_{e}, \mathbf{p}_{e}, t\right)
$$

where $\mathbf{q}_{e}(t)=\left[q_{1 e}(t), q_{2 e}(t), \ldots, q_{n e}(t)\right]^{\mathrm{T}}$ contains the time series of the $n$ independent states, and $\mathbf{p}_{e}$ are the parameters corresponding to the experimental system. It will be assumed that the experimental measurements $\mathbf{y}_{e}(t)=\mathbf{q}_{e}(t)+\eta(t)$ are corrupted by zero-mean noise $\eta(t)=\left[\eta_{1}(t), \eta_{2}(t), \ldots, \eta_{n}(t)\right]^{\mathrm{T}}$ and are available over the time interval $t=\left[0, t_{f}\right]$. The corresponding mathematical model that will be used for identification purposes is of the following general form:

$$
\dot{\mathbf{q}}=\mathbf{f}(\mathbf{q}, \mathbf{p}, t),
$$

where $\mathbf{q}(t)=\left[q_{1}(t), q_{2}(t), \ldots, q_{n}(t)\right]^{\mathrm{T}}$ are the corresponding states and $\mathbf{p}=\left[p_{1}, p_{2}, \ldots, p_{\ell}\right]^{\mathrm{T}}$ are the parameters to be identified. The goal is to determine the parameter vector $\mathbf{p}$ that minimizes the error between the predicted response $\mathbf{q}(t)$ and the experimental measurements $\mathbf{y}_{e}(t)$ :

$$
J(\mathbf{p})=\frac{1}{2} \int_{0}^{t_{f}}[\mathbf{e}(\mathbf{p}, t)+\eta(t)]^{\mathrm{T}}[\mathbf{e}(\mathbf{p}, t)+\eta(t)] d t,
$$

where $J(\mathbf{p})$ is the objective function to be minimized, and $\mathbf{e}(\mathbf{p}, t)=\left[e_{1}(\mathbf{p}, t), e_{2}(\mathbf{p}, t), \ldots, e_{n}(\mathbf{p}, t)\right]^{\mathrm{T}}$ is the error vector, whose $k^{\text {th }}$ entry is defined as $e_{k}(t)=q_{k e}(t)-q_{k}(\mathbf{p}, t)$. Unmeasured states have zero error; if experimental data are available for only one state, the error vector is simply $\mathbf{e}(t)=$ $\left[e_{1}(\mathbf{p}, t), 0, \ldots, 0\right]^{\mathrm{T}}$. In general, $J(\mathbf{p})$ will contain many local minima; the mathematical model (3) that is most representative of the experimental system (2) is obtained when the parameters p correspond to the global minimum of the above objective function (4).

To find the global minimum of $J(\mathbf{p})$, a homotopy transformation is introduced into the objective function:

$$
J(\mathbf{p}, \lambda)=\frac{1}{2} \int_{0}^{t_{f}}[\mathbf{e}(\mathbf{p}, \lambda, t)+\eta(t)]^{\mathrm{T}}[\mathbf{e}(\mathbf{p}, \lambda, t)+\eta(t)] d t,
$$

which can be expanded as follows:

$$
\begin{aligned}
J(\mathbf{p}, \lambda)= & \frac{1}{2} \int_{0}^{t_{f}}\left[\mathbf{e}(\mathbf{p}, \lambda, t)^{\mathrm{T}} \mathbf{e}(\mathbf{p}, \lambda, t)\right] d t \\
& +\int_{0}^{t_{f}}\left[\mathbf{e}(\mathbf{p}, \lambda, t)^{\mathrm{T}} \boldsymbol{\eta}(t)\right] d t+\frac{1}{2} \int_{0}^{t_{f}}\left[\boldsymbol{\eta}(t)^{\mathrm{T}} \boldsymbol{\eta}(t)\right] d t .
\end{aligned}
$$

Since the shape of $J(\mathbf{p}, 0)$ is unknown, the homotopy transformation is introduced into the mathematical model (3), not directly into the objective function:

$$
\dot{\mathbf{q}}=\mathbf{f}(\mathbf{q}, \mathbf{p}, t)+\lambda \boldsymbol{\Gamma} \mathbf{e}(t)+\lambda \boldsymbol{\Gamma} \eta(t) .
$$

The last two terms in (7) are related to the use of observers in dynamical systems [11], with gain matrix $\boldsymbol{\Gamma}$.

To study the influence of the homotopy terms on the objective function, (7) is subtracted from (2), whereupon the following equation governing the dynamics of the error is obtained:

$$
\dot{\mathbf{e}}(t)+\lambda \boldsymbol{\Gamma} \mathbf{e}(t)=\mathbf{f}\left(\mathbf{q}_{e}, \mathbf{p}_{e}, t\right)-\mathbf{f}(\mathbf{q}, \mathbf{p}, t)-\lambda \boldsymbol{\Gamma} \eta(t) .
$$

Equation (8) is similar to that governing the dynamics of a damped first-order system, and has a static equilibrium at $\mathbf{e}(t)=\mathbf{0}$ when $\mathbf{p}=\mathbf{p}_{e}$. Assuming the initial conditions $\mathbf{q}(0)=\mathbf{q}_{e}(0)$ are used for the mathematical model, the initial conditions of the error system are $\mathbf{e}(0)=\mathbf{0}$. By choosing an appropriate gain matrix $\boldsymbol{\Gamma}$, the effect of the forcing terms in (8) can be reduced. In other words, by increasing the stiffness $(\boldsymbol{\Gamma})$ of the first-order system (8), it is forced to oscillate very close to its equilibrium. Since the amplitude of the noise term $\lambda \Gamma \eta(t)$ in (8) increases as the entries in gain matrix $\Gamma$ increase, $\Gamma$ should be selected such that the synchronization error (4) is as small as possible when using the initial parameter guesses in the mathematical model. For a given noise signal $\boldsymbol{\eta}(t)$, there is an optimal $\boldsymbol{\Gamma}$ at which the best possible synchronization occurs; in the absence of noise, the synchronization improves as $\boldsymbol{\Gamma}$ (the stiffness) is increased.

The properties of the error system (8) can be further examined by expanding $\mathbf{q}$ around $\mathbf{q}_{e}$ and $\mathbf{p}$ around $\mathbf{p}_{e}$ :

$$
\dot{\mathbf{e}}(t)+\left[\lambda \boldsymbol{\Gamma}-\frac{\partial \mathbf{f}}{\partial \mathbf{q}_{e}}\right] \mathbf{e}(t) \approx \frac{\partial \mathbf{f}}{\partial \mathbf{p}_{e}} \delta \mathbf{p}-\lambda \boldsymbol{\Gamma} \boldsymbol{\eta}(t),
$$

where $\delta \mathbf{p}=\mathbf{p}_{e}-\mathbf{p}$ and the higher-order terms have been neglected. Note that, for a given value of $\lambda$, the error signal $\mathbf{e}(t)$ will be stable provided the eigenvalues of the matrix $-\left[\lambda \boldsymbol{\Gamma}-\partial \mathbf{f} / \partial \mathbf{q}_{e}\right]$ are negative. With a suitable choice of $\boldsymbol{\Gamma}$, the solution of the singularly perturbed system (9) will exhibit a boundary layer effect [23], where the deviation of the mathematical model from the experimental data vanishes on a fast time scale, and the solution on a slow time scale $(\dot{\mathbf{e}}(t)=\mathbf{0})$ behaves as follows:

$$
\begin{aligned}
\mathbf{e}(t)= & {\left[\lambda \boldsymbol{\Gamma}-\frac{\partial \mathbf{f}}{\partial \mathbf{q}_{e}}\right]^{-1} \frac{\partial \mathbf{f}}{\partial \mathbf{p}_{e}} \delta \mathbf{p} } \\
& -\lambda\left[\lambda \boldsymbol{\Gamma}-\frac{\partial \mathbf{f}}{\partial \mathbf{q}_{e}}\right]^{-1} \boldsymbol{\Gamma} \boldsymbol{\eta}(t) \triangleq \mathbf{A} \delta \mathbf{p}-\mathbf{b} .
\end{aligned}
$$

Left-multiplying both sides of (10) by $\mathbf{e}(t)^{\mathrm{T}}$ and integrating over the time interval $t=\left[0, t_{f}\right]$, we obtain the following:

$$
\begin{aligned}
\int_{0}^{t_{f}}\left[\mathbf{e}(t)^{\mathrm{T}} \mathbf{e}(t)\right] d t= & \delta \mathbf{p}^{\mathrm{T}}\left[\int_{0}^{t_{f}} \mathbf{A}^{\mathrm{T}} \mathbf{A} d t\right] \delta \mathbf{p}+\int_{0}^{t_{f}}\left[\mathbf{b}^{\mathrm{T}} \mathbf{b}\right] d t \\
& -2 \delta \mathbf{p}^{\mathrm{T}} \int_{0}^{t_{f}}\left[\mathbf{A}^{\mathrm{T}} \mathbf{b}\right] d t
\end{aligned}
$$

Both sides of (10) are now left-multiplied by $\eta(t)^{\mathrm{T}}$ and integrated over the time interval $t=\left[0, t_{f}\right]$ :

$$
\int_{0}^{t_{f}}\left[\boldsymbol{\eta}(t)^{\mathrm{T}} \mathbf{e}(t)\right] d t=\delta \mathbf{p}^{\mathrm{T}} \int_{0}^{t_{f}} \mathbf{c} d t-\int_{0}^{t_{f}} \mathbf{g} d t,
$$


where $\mathbf{c}=\mathbf{A}^{\mathrm{T}} \boldsymbol{\eta}(t)$ and $\mathbf{g}=\boldsymbol{\eta}(t)^{\mathrm{T}} \mathbf{b}$. Substituting (11) and (12) into (6), we obtain the following:

$$
\begin{aligned}
J(\mathbf{p}, \lambda)= & \frac{1}{2} \delta \mathbf{p}^{\mathrm{T}}\left[\int_{0}^{t_{f}} \mathbf{A}^{\mathrm{T}} \mathbf{A} d t\right] \delta \mathbf{p} \\
& +\delta \mathbf{p}^{\mathrm{T}}\left[\int_{0}^{t_{f}}\left(\mathbf{c}-\mathbf{A}^{\mathrm{T}} \mathbf{b}\right) d t\right]+C,
\end{aligned}
$$

where $\quad C=\frac{1}{2} \int_{0}^{t_{f}}\left[\mathbf{b}^{\mathrm{T}} \mathbf{b}\right] d t-\int_{0}^{t_{f}} \mathbf{g} d t+\frac{1}{2} \int_{0}^{t_{f}}\left[\eta(t)^{\mathrm{T}} \eta(t)\right] d t$, which is not a function of $\delta \mathbf{p}$.

From (13), it can be concluded that the objective function is quadratic in the parameter error $\delta \mathbf{p}$ provided that gain matrix $\boldsymbol{\Gamma}$ is selected so as to ensure the synchronization [12] of the mathematical model to the experimental data [i.e., (10) holds]. The value of $\mathbf{p}^{*}$ at which the objective function $J(\mathbf{p}, \lambda)$ attains a minimum can be expressed as follows:

$$
\mathbf{p}^{*}=\mathbf{p}_{e}+\left[\int_{0}^{t_{f}} \mathbf{A}^{\mathrm{T}} \mathbf{A} d t\right]^{-1} \int_{0}^{t_{f}}\left(\mathbf{c}-\mathbf{A}^{\mathrm{T}} \mathbf{b}\right) d t .
$$

In the absence of noise [i.e., if $\boldsymbol{\eta}(t)=\mathbf{0}$ ], the homotopy terms do not shift the location of the global minimum (i.e., $\left.\mathbf{p}^{*}=\mathbf{p}_{e}\right)$; in the presence of noise, the optimal parameters are shifted by the amount shown in (14). Two numerical examples are presented below to illustrate the application of the single-shooting homotopy method for parameter identification in dynamical systems.

\section{NUMERICAL EXAMPLES}

The Lorenz oscillator is first considered to illustrate the effect of homotopy terms on the shape of the objective function. In its original form, the Lorenz system is defined as follows:

$$
\begin{aligned}
& \dot{q}_{1 e}=-p_{1 e} q_{1 e}+p_{1 e} q_{2 e}, \\
& \dot{q}_{2 e}=p_{2 e} q_{1 e}-q_{2 e}-q_{1 e} q_{3 e}, \\
& \dot{q}_{3 e}=q_{1 e} q_{2 e}-p_{3 e} q_{3 e} .
\end{aligned}
$$

This system is chaotic when the parameters $p_{1 e}=10, p_{2 e}=$ 28 , and $p_{3 e}=8 / 3$ are used. The initial conditions for the experimental system are $q_{1 e}(0)=1.2, q_{2 e}(0)=2.1$, and $q_{3 e}(0)=1.7$. The following mathematical model is now used to identify parameters $p_{1}, p_{2}$, and $p_{3}$ :

$$
\begin{aligned}
& \dot{q}_{1}=-p_{1} q_{1}+p_{1} q_{2}+\lambda \gamma_{1}\left(q_{1 e}+\eta_{1}-q_{1}\right), \\
& \dot{q}_{2}=p_{2} q_{1}-q_{2}-q_{1} q_{3}+\lambda \gamma_{1}\left(q_{1 e}+\eta_{1}-q_{1}\right), \\
& \dot{q}_{3}=q_{1} q_{2}-p_{3} q_{3}+\lambda \gamma_{1}\left(q_{1 e}+\eta_{1}-q_{1}\right)
\end{aligned}
$$

where (16) has been augmented with homotopy transformation terms corresponding to a gain matrix $\boldsymbol{\Gamma}$ consisting of three identical rows of $[20,0,0]$. Note that the original system $(15)$ is recovered upon substitution of $\lambda=0$ into the augmented system (16). As suggested by (16), it is assumed that only $y_{1 e}(t)=$ $q_{1 e}(t)+\eta_{1}(t)$ is measured, where $\eta_{1}(t) \sim \mathcal{N}(0,0.25) .{ }^{1}$ A $10-\mathrm{s}$ time series of the experimental system (15) is generated, which

\footnotetext{
${ }^{1}$ The noise is Gaussian with zero mean and a variance of 0.25 .
}



FIG. 1. (Color online) Evolution of parameter estimates for the Lorenz system, normalized relative to experimental values.

is used to evaluate the following objective function during the minimization process: ${ }^{2}$

$$
J=\sum_{i=1}^{k}\left\{\int_{0}^{t_{f}}\left(q_{i e}(t)+\eta_{i}(t)-q_{i}\left({ }^{\lambda} p_{1},{ }^{\lambda} p_{2},{ }^{\lambda} p_{3}, t\right)\right)^{2} d t\right\},
$$

where $k=1$ and $t_{f}=10$. The following noise-corrupted initial conditions are used for the mathematical model (16): $q_{1}(0)=1.5570, q_{2}(0)=2.2533$, and $q_{3}(0)=2.1952$; the initial parameter guesses at $\lambda=1$ are chosen as ${ }^{1} p_{1}^{0}=20$, ${ }^{1} p_{2}^{0}=38$, and ${ }^{1} p_{3}^{0}=12.67$. The evolution of the parameter estimates during the optimization process is shown in Fig. 1 for decreasing values of $\lambda .^{3}$ The final identified parameters

\footnotetext{
${ }^{2}$ In both examples, the optimization is performed using the fminsearch function in MATLAB, which is based on the Nelder-Mead simplex method [24]. The differential equations are solved using the ode 45 variable-time-step integrator with absolute and relative error tolerances of $10^{-6}$. Since measurement data are collected every $10 \mathrm{~ms}$, the integral in the objective function is replaced with discrete summation. The noisy experimental data are linearly interpolated during the numerical integration to accommodate the use of a variable-time-step integrator.

${ }^{3} \lambda$ is decreased in increments of 0.25 . At each value of $\lambda, 25$ iterations of fminsearch are performed, each of which corresponds to at least one simulation of (16) (function call).
}

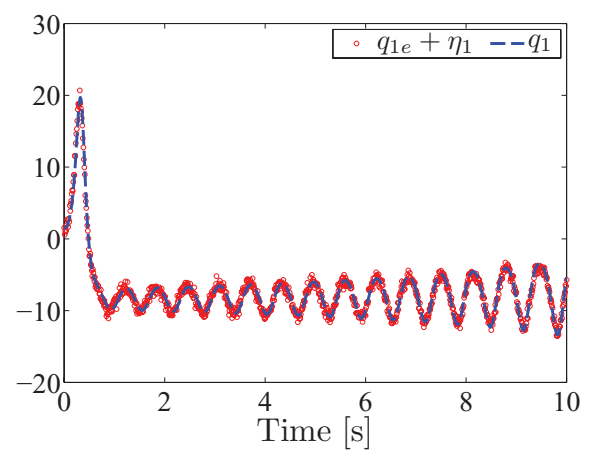

FIG. 2. (Color online) Experimental measurement $\left(q_{1 e}+\eta_{1}\right)$ and predicted response using identified parameters $\left(q_{1}\right)$ for the Lorenz system. 



FIG. 3. (Color online) Evolution of parameter estimates for the Rössler system, normalized relative to experimental values.

are ${ }^{0} p_{1}^{*}=10.7311,{ }^{0} p_{2}^{*}=28.3848$, and ${ }^{0} p_{3}^{*}=2.6030$, which result in excellent agreement between the experimental system and the mathematical model, as shown in Fig. 2. Note that, despite using noisy initial conditions for the mathematical model, the predicted response lies within the envelope of the noisy experimental measurement. The slight deviation of the identified parameters from the experimental parameters compensates for the small error in the initial conditions. The exact parameters are identified when the value of $\lambda$ is nearly (but not exactly) zero. The homotopy term with a small value of $\lambda$ must be retained if the experimental data are collected over a long time interval. If the homotopy term is absent $(\lambda=0)$, the model response will eventually deviate from the experimental data due to the nature of chaotic systems. The smallest value of $\lambda$ that must be retained is problem-specific, and will depend on the amplitude and nature of the noise in the experimental data. Some guidelines for selecting the smallest suitable value of $\lambda$ are suggested in the work of Abarbanel et al. [3].

The Rössler system is now considered:

$$
\begin{aligned}
& \dot{q}_{1 e}=-q_{2 e}-q_{3 e}, \\
& \dot{q}_{2 e}=q_{1 e}+p_{1 e} q_{2 e} \\
& \dot{q}_{3 e}=p_{2 e}+q_{1 e} q_{3 e}-p_{3 e} q_{3 e} .
\end{aligned}
$$

The parameters used for generating the experimental data are $p_{1 e}=p_{2 e}=0.2$ and $p_{3 e}=5.7$; the initial conditions are $q_{1 e}(0)=q_{2 e}(0)=q_{3 e}(0)=3$. It shall be assumed that $y_{1 e}=q_{1 e}+\eta_{1}, y_{2 e}=q_{2 e}+\eta_{2}$, and $y_{3 e}=q_{3 e}+\eta_{3}$ are measured over the time interval $t=[0,25]$. The following mathematical model is used to identify parameters $p_{1}, p_{2}$, and $p_{3}$ :

$$
\begin{aligned}
& \dot{q}_{1}=-q_{2}-q_{3}+\lambda \gamma_{1}\left(q_{1 e}+\eta_{1}-q_{1}\right), \\
& \dot{q}_{2}=q_{1}+p_{1} q_{2}+\lambda \gamma_{2}\left(q_{2 e}+\eta_{2}-q_{2}\right), \\
& \dot{q}_{3}=p_{2}+q_{1} q_{3}-p_{3} q_{3}+\lambda \gamma_{3}\left(q_{3 e}+\eta_{3}-q_{3}\right),
\end{aligned}
$$
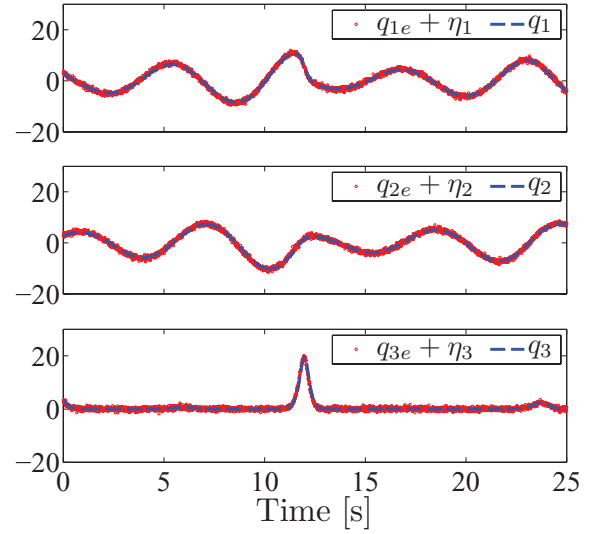

FIG. 4. (Color online) Experimental measurements $\left(q_{i e}+\eta_{i}\right)$ and predicted responses using identified parameters $\left(q_{i}\right)$ for the Rössler system.

where $\gamma_{1}=\gamma_{2}=\gamma_{3}=10$ and the noisy initial conditions $q_{1}(0)=2.9881, q_{2}(0)=2.6808$, and $q_{3}(0)=3.2613$ are used. An objective function of the same form as (17) is minimized, where $k=3, t_{f}=25$, and $\eta_{i}(t) \sim \mathcal{N}(0,0.25)$ in this case. The evolution of the parameter estimates during the optimization process is shown in Fig. 3 for decreasing values of $\lambda$. Note that the parameters converge at different values of $\lambda$ as new valleys are formed in the parameter space of the objective function, which illustrates the utility of the homotopy approach. The final identified parameters are ${ }^{0} p_{1}^{*}=0.2099,{ }^{0} p_{2}^{*}=0.2164$, and ${ }^{0} p_{3}^{*}=5.5838$, which again result in excellent agreement between the experimental system and the mathematical model, as shown in Fig. 4.

\section{CONCLUSIONS}

In this paper, we have presented a method for identifying parameters in mathematical models where the explicit functional form of the equations is known exactly. We have explicitly demonstrated that the objective function becomes quadratic upon the addition of the homotopy terms in the mathematical model. Since homotopy optimization can be used with singleshooting algorithms, the more complicated multiple-shooting techniques can be avoided. The above analysis and numerical examples involving chaotic systems suggest that the proposed method is an effective strategy for identifying parameters in dynamical systems.

\section{ACKNOWLEDGMENTS}

C.P.V. acknowledges the financial support received from the Ontario Centres of Excellence. T.U. and J.M. gratefully acknowledge the financial support provided by the Natural Sciences and Engineering Research Council of Canada (NSERC), and the NSERC/Toyota/Maplesoft Industrial Research Chair program.
[1] H. D. I. Abarbanel, R. Brown, J. J. Sidorowich, and L. S. Tsimring, Rev. Mod. Phys. 65, 1331 (1993).
[2] D. Brewer, M. Barenco, R. Callard, M. Hubank, and J. Stark, Philos. Trans. R. Soc. London A 366, 519 (2008). 
[3] H. D. I. Abarbanel, D. R. Creveling, R. Farsian, and M. Kostuk, SIAM J. Appl. Dyn. Syst. 8, 1341 (2009).

[4] D. R. Creveling, P. E. Gill, and H. D. I. Abarbanel, Phys. Lett. A 372, 2640 (2008).

[5] A. Maybhate and R. E. Amritkar, Phys. Rev. E 59, 284 (1999).

[6] U. Parlitz, Phys. Rev. Lett. 76, 1232 (1996).

[7] U. Parlitz, L. Junge, and L. Kocarev, Phys. Rev. E 54, 6253 (1996).

[8] C. A. Floudas, Deterministic Global Optimization: Theory, Methods and Applications (Kluwer Academic Publishers, Dordrecht, 2000).

[9] G. L. Baker and J. P. Gollub, Chaotic Dynamics: An Introduction, 2nd ed. (Cambridge University Press, Cambridge, 1996).

[10] S. Chen, J. Hu, C. Wang, and J. Lü, Phys. Lett. A 321, 50 (2004).

[11] H. Nijmeijer and I. M. Y. Mareels, IEEE Trans. Circuits Syst. I 44, 882 (1997).

[12] C. Letellier and L. A. Aguirre, Phys. Rev. E 82, 016204 (2010).

[13] H. D. I. Abarbanel, D. R. Creveling, and J. M. Jeanne, Phys. Rev. E 77, 016208 (2008).

[14] E. Baake, M. Baake, H. G. Bock, and K. M. Briggs, Phys. Rev. A 45, 5524 (1992).
[15] Z. Jia-fan, Y. Qing-hua, and Z. Tong, ASME J. Dyn. Syst. Meas. Control 133, 051002 (2011).

[16] J.-F. Chang, Y.-S. Yang, T.-L. Liao, and J.-J. Yan, Expert Syst. Appl. 35, 2074 (2008).

[17] C. Tao, Y. Zhang, and J. J. Jiang, Phys. Rev. E 76, 016209 (2007).

[18] D. M. Dunlavy, D. P. O’Leary, D. Klimov, and D. Thirumalai, J. Comput. Biol. 12, 1275 (2005).

[19] L. T. Watson and C. Y. Wang, Int. J. Solids Struct. 17, 29 (1981).

[20] L. T. Watson and R. T. Haftka, Comput. Methods Appl. Mech. Eng. 74, 289 (1989).

[21] C. P. Vyasarayani, T. Uchida, A. Carvalho, and J. McPhee, Multibody Syst. Dyn. 26, 411 (2011).

[22] C. P. Vyasarayani, T. Uchida, and J. McPhee, ASME J. Comput. Nonlin. Dyn. 7, 011012 (2012).

[23] J. A. Sanders, F. Verhulst, and J. Murdock, Averaging Methods in Nonlinear Dynamical Systems, 2nd ed. (Springer, New York, 2007).

[24] M. Avriel, Nonlinear Programming: Analysis and Methods (Dover Publications, Mineola, 2003). 